\title{
VICO \\ A Tool for Supporting Visual Comparisons of Different Pine-Beetle Management Approaches
}

\author{
Angela Cristina Duta, M. S. T. Carpendale, Ken Barker \\ Department of Computer Science \\ University of Calgary \\ \{mituac, sheelagh, barker\}@cpsc.ucalgary.ca
}

\begin{abstract}
In this paper a visual tool for comparing different mountain pine-beetle management approaches is presented. This comparison tool gives the users the possibility of viewing their information, but more importantly it provides the ability to visually see the similarities and the differences between two simulations.

This tool can help users to make decisions regarding their future actions against beetle attacks. The technique used is based on colour indexing combined with the mapping of the strategies into colour histograms. The system is entitled VICO from the phrase Visual Comparison.
\end{abstract}

Keywords: scenario, visual comparison between maps, colour histogram, probability, simulation.

\section{Introduction}

Each year the mountain pine beetle destroys millions of mature mountain pine trees in British Columbia. Unfortunately these epidemics have attacked many forest resources such as the Banff National Park. The Ministry of Forests [9] has coordinated the development of a number of management approaches in order to stop the killing of pines by these destructive insects.

The Kamloops Landscape Model (KLM) [5, 6] was created in order to evaluate the results of mountain pine beetle (MPB) management activities in Kamloops forest districts. Two aspects were taken into consideration during the evaluation: the influence on the volume of timber from the infested area and the growth of the beetle population. The main goal of MPB management activities was to increase the volume of timber that can still be used in the forest industry. This can be achieved by either decreasing the volume of timber killed by beetles or salvaging the trees that have been already attacked.

The MPB management activities combine four different techniques:
- Clearcut harvesting of beetle infested areas.

- Harvesting of susceptible blocks.

- Salvage of the attacked blocks.

- Single tree treatments: fell and burn or spread of MSMA.

The MSMA method permits the recuperation of the standing dead wood. Fell and burn are just used to prevent the spread of infestation.

The KLM approach showed that timber from areas with no MPB management is more likely to be destroyed by MPB than timber from those areas where MPB management is used to prevent beetles from spreading. However, this is not a uniform response. The increase in the effort of fighting against beetles does not always result in a higher benefit. The KLM model showed that a decrease in beetle management in areas affected by beetle infections has as a result an increase in MPB attacks. On the other hand sometimes an increase in beetle management determines a marginal increase in benefits.

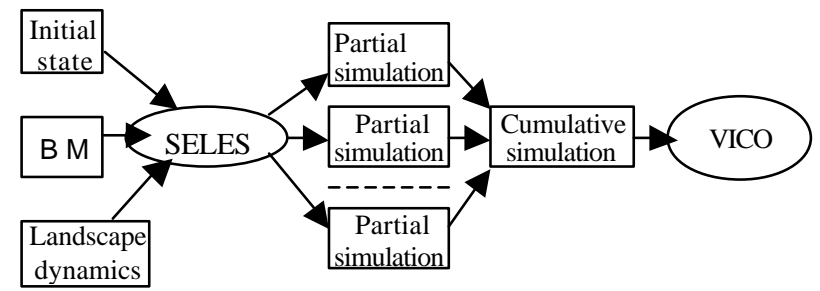

Figure 1: The relationship between SELES and VICO

The SELES (Spatially Explicit Landscape Event Simulator) $[4,6,10]$ is a tool for simulating the landscape dynamics that has been used for studying the areas affected by MPB attacks. SELES is a stochastic environment working with various assumptions like the rate of the beetle growth, the temperature over the periods of a year, accidental strikes of light and fire, precipitations 
etc., also called landscape dynamics. Since SELES is stochastic it runs ten Monte Carlo simulations for each scenario and then cumulates them as a final output. These ten simulations will be referred as partial simulations, while their combined result as a cumulative simulation. The practice of generating ten partial simulations combined in a cumulative one is called a simulation run

The VICO project aims to create a tool to facilitate choosing the most suitable landscape ecology management for any area already infected by beetles. The goal of this project is to provide users with a tool that helps them to visually compare cumulative simulations. By offering easy to understand visual comparison tools for data generated by SELES simulations, VICO makes a powerful assistant for making decisions about fighting against MPB attacks (see Figure 1).

Other terms used in this paper are strategy and scenario. A strategy or a beetle management approach means a group of actions performed together in order to stop the beetle attacks. A scenario refers to a strategy that can be applied to a particular location at a particular time and the probable result to be obtained.

\section{SELES}

SELES [4,6,10] models and simulates landscape dynamics. A landscape is a heterogeneous region that generally is composed differing areas with differing interacting ecosystems. Analyses of landscape change tend to involve great distances and tend to take place over long periods of time. This makes experimentation impractical and leads to a need for the development of landscape models. These models can be of use in predicting the consequences of management actions.

\section{Related work}

Histograms are frequently used for the presentation of information. They have also been used to recognise individual objects. Swain and Ballard [8] have developed an image search machine using histograms to search for an object in a database of images. The first step in searching is to create a histogram for each image. Each histogram shows the number of pixels for every colour that appears in the image. The second step is to distinguish objects from their background and redraw each histogram according to objects found into the image. The third step is to compare the histogram of the query object with the histograms of images. Sometimes the query object appears in another image having other dimensions and because of that it is difficult to detect its presence.

Pass and Zabih [7] have extended this method by using a joint histogram instead of just a simple colour one. A joint histogram contains information about a group of pixels according to a set of features like RGB colours, edge density, degree of texture, rank and gradient magnitude. This method gives better results even when searching in totally different images that have similar colour histograms.

"Descartes" (earlier called "IRIS") [1, 2] is an application developed to compare values of certain statistical indices regarding several countries at a particular moment in time. It does not use histograms, but a bar according to the statistical index is drawn in the area of each country considered for comparison.

Visualising data generated by SELES was the goal of another tool called "Tardis"[3]. Tardis is an extension of SELES and it is focused on 3-dimensional visualisation of simulations generated by SELES. Tardis' main goal is to show the spatial-temporal changes that occur in landscape dynamics. One visualisation included in the Tardis is like a movie. One can move through it from the beginning to the end, or one can go directly to the end of it to see the final scene or one can stop it at time in the simulation and move either forward or backward. This allows one to see the simulated landscape situated both spatially and temporally (see Figure 2).

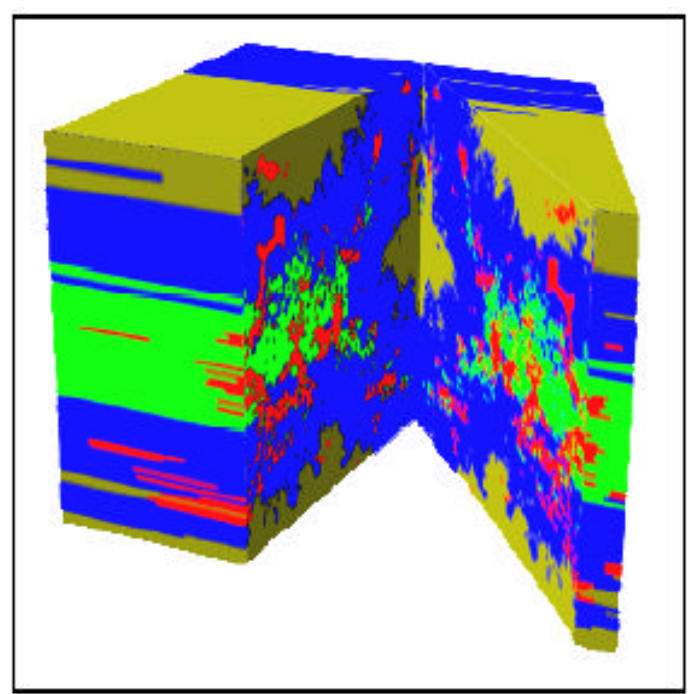

Figure 2: Tardis: visualization of the spatialtemporal "movie" [3]

\section{Comparison between scenarios}

Four scenarios have been identified to fight against beetles, scenarios that have varying levels of pine beetle management: Beetle Management (BM), BM*2, BM/2 and $\mathrm{BM}^{*} 10$. Their names express the effort involved in each strategy compared to the first one (BM), not necessarily the results obtained.

It is not easy to decide which scenario is more efficient at a particular moment in time for a specific area 
due to different species of trees found in that area, different bio-geo-climatic influences and the elevation of the cost of implementing scenarios can be very different. An interesting set of terms for comparison is:

- Cost of the beetle management

- Effect on beetle population

- Amount of wood lost / salvaged

- Size of infestation area

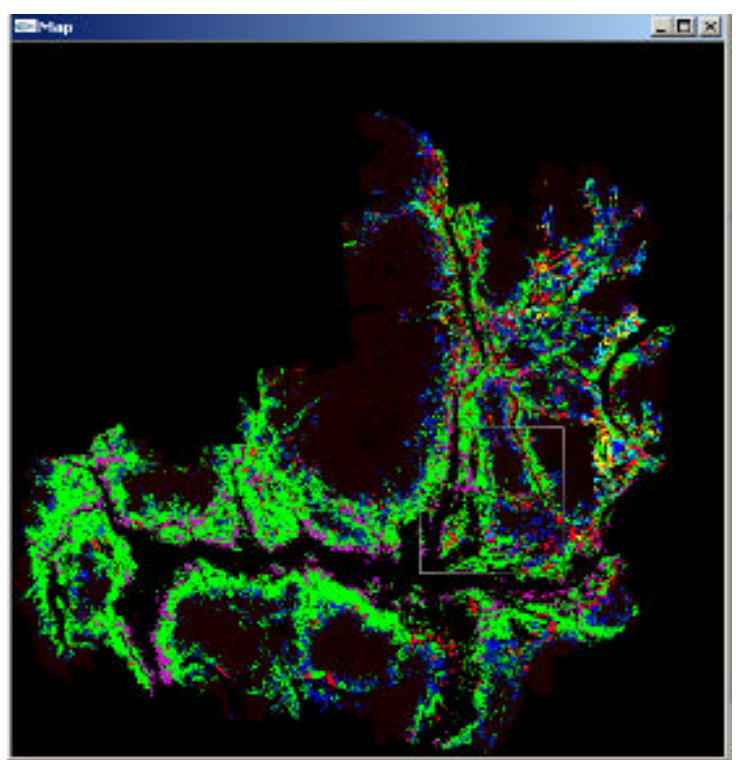

Figure 3: An inventory map showing tree types

VICO (VIsual COmparison) is a tool developed to permit comparison between different simulations generated by SELES (see Figure 1) in terms of the area of infestation. The user can identify exactly the areas that will be most likely affected by beetle attacks depending on the strategy applied. At the beginning, a map of a specific region is chosen where there is already a history of the activity of mountain pine beetles. The terrain data used to develop the comparison model was obtained from the Canadian Forest Service and British Columbia Ministry of Forest. The starting map representing an area attacked by MPB is one of the following:

- A bio-geo-climatic map of the area

- An inventory map showing the types of trees growing in that area (see Figure 3)

- An elevation map in metres

- A map showing the starting beetle population In VICO the user can select two scenarios to compare. They can be:

- Two cumulative simulations of different MPB approaches for the same period of time.

- Two cumulative simulations of the same MPB approach for two different periods of time. the area. Another aspect to take into consideration is that

- Two cumulative simulations of different MPB approaches for two different periods of time.

Each cumulative simulation of a scenario results in a map that is created by adding the results of running the simulation ten times. It is a map of probabilities of beetle attacks, where probabilities are visually represented using different colours. As each of the partial simulations based on probabilities it can result a different map of beetle attacks in an area. Table 1 shows the signification of colours in the cumulative simulation.

Table 1: The meaning of colours in cumulative simulations

\begin{tabular}{|c|c|}
\hline Colour & $\begin{array}{c}\text { Probability to be } \\
\text { attacked } \\
\text { (out of 10) }\end{array}$ \\
\hline Black & 0 \\
\hline Green & 1 \\
\hline Cyan & 2 \\
\hline Light blue & 3 \\
\hline Blue & 4 \\
\hline Dark blue & 5 \\
\hline Pink & 6 \\
\hline Light red & 7 \\
\hline Dark red & 8 \\
\hline Orange & 9 \\
\hline Yellow & 10 \\
\hline
\end{tabular}

The probability that a cell is attacked is the number of partial simulations in the cumulative simulation, in which that cell is simulated as being attacked.
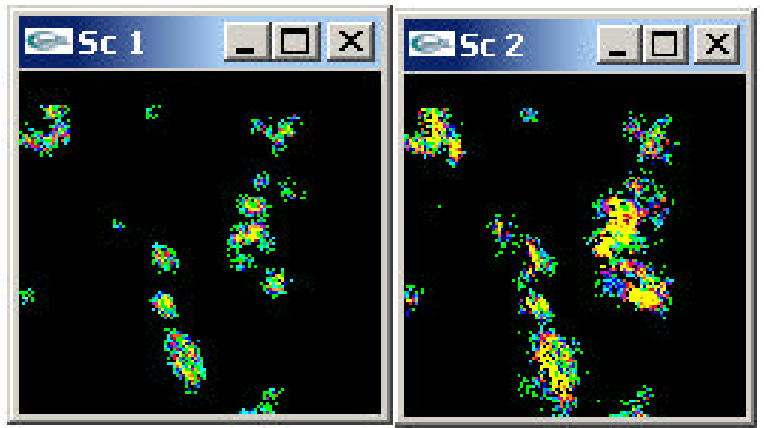

Figure 4: Two cumulative simulations showing the probable MPB attacks in the same area. The first one is showing the area after BM has been applied for one year and the second one BM/2 after ten years 


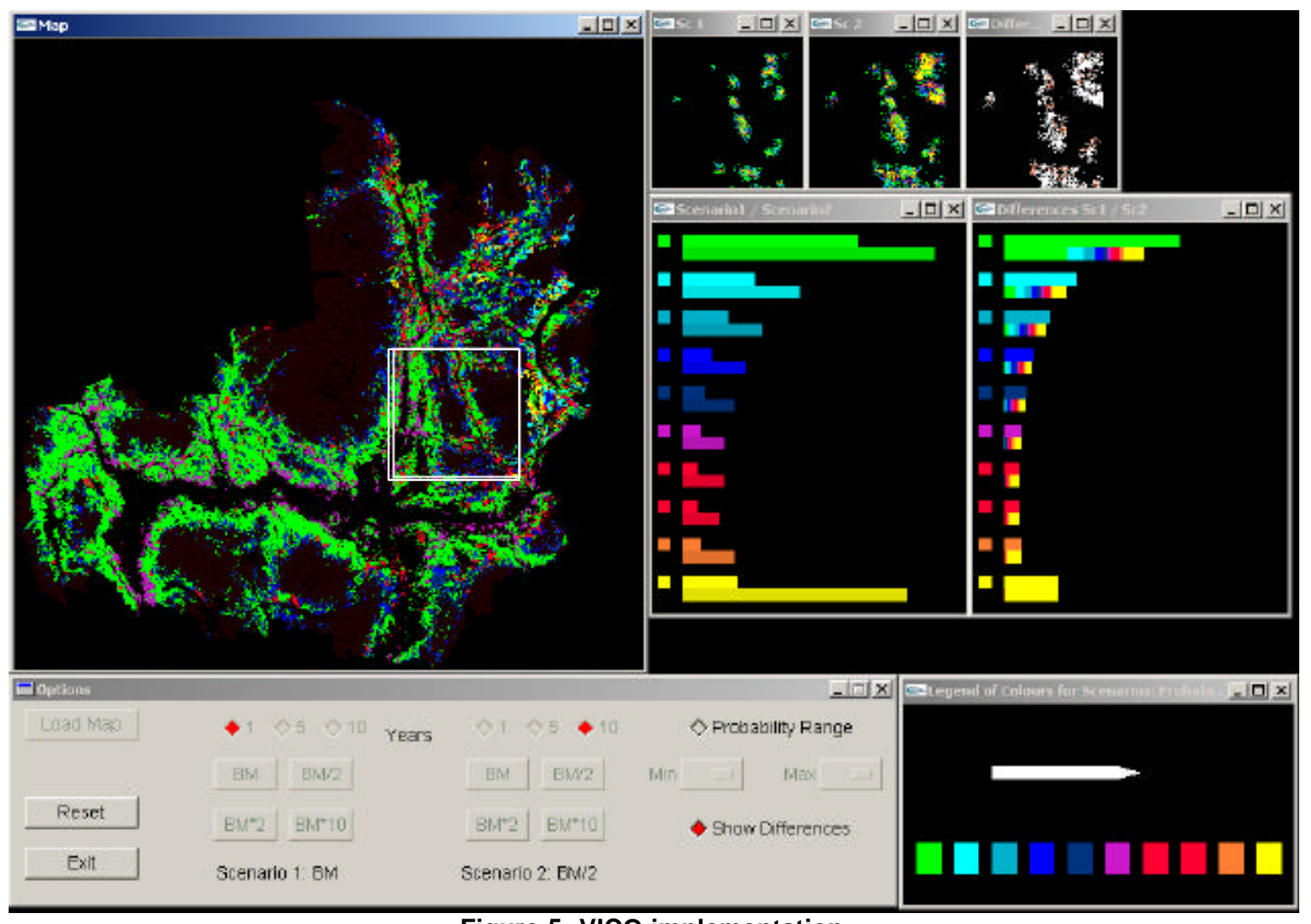

Figure 5: VICO implementation

\section{How does VICO work?}

In VICO (see Figure 5) the user has the possibility of choosing the type of map he/she wants: a bio-geo-climatic map of the area, an inventory map showing the types of trees growing in that area, an elevation map or a map showing the starting beetle population or even a map of another cumulative simulation. Then the user has to choose the type of beetle management strategy and the number of years the simulation should be run. For example: the first scenario can be BM showing the cells attacked after one year from the moment when the beetle attack was recorded and the second one can be BM/2 applied for ten years (see Figure 4).

\subsection{The comparison histogram}

After selecting the two scenarios two small windows will appear to show parts of the cumulative simulations according to the parts selected from the map (see Figure 4). The user can compare these two scenarios by comparing parts of them displayed in two small windows. To facilitate the comparison another window displays a histogram of the numbers of cells probable to be attacked in each scenario. The histogram is drawn according to the area of the map that is currently under the observation.

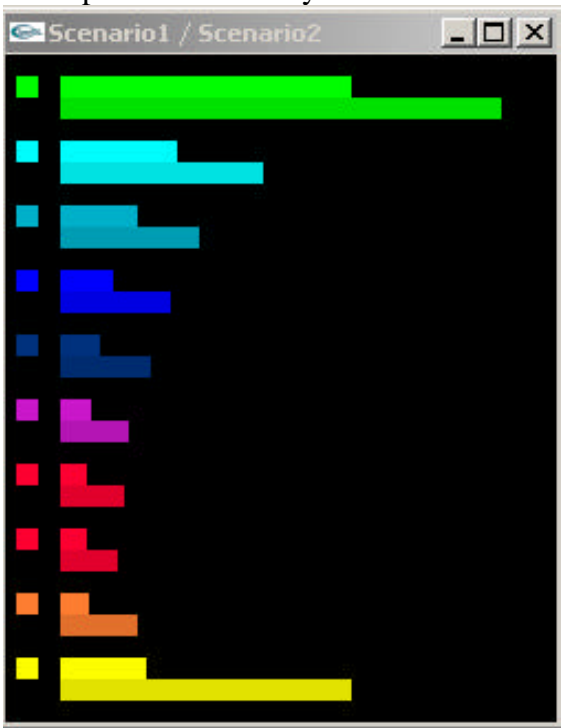

Figure 6: The histogram for comparison 
The bars drawn represent relative values of the selected area from the map. Every time a small area is selected the numbers of cells from each scenario are computed according to their probability. Then the maximum of these numbers is chosen and all the values are divided by this maximum. This method ensures a good visual representation every time the histogram is redrawn. The position of bars, one under the other, grouped by the values of probability, makes differences easy to see. The small coloured squares from the left side of the window show all the possible probabilities for a cell to be attacked. In case in one scenario or in both scenarios there are no cells to be attacked with a certain probability the small square from the left side of the histogram will just let the user know that (see Figure 6).

\subsection{Cells within a range of probability}

The user may be interested only in the comparison of the cells attacked with the probability in a certain range, for example: either lower than $50 \%$, or more than $50 \%$, or between $30 \%$ and $60 \%$ (see Figure 7). For this a filter specifying the upper value and the lower value of the probability considered is incorporated in the application.

Feset

Figure 7: Options in VICO

\subsection{Seeing the differences}

In order to depict the differences between two scenarios in a detailed manner the user can choose to display another window (see Figure 8). Two colours appear in it:

- white for cells where applying scenario 1 there is a lower probability to be infected.

- brown for cells where applying scenario 2 there is a lower probability to be infected.

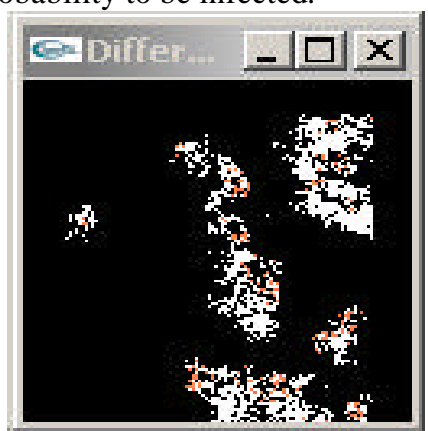

Figure 8: Window showing differences between two scenarios

\subsection{The histogram for differences}

Another histogram is attached to the window of differences showing in a visual way the differences between scenarios. The starting point for this histogram refers to the numbers of cells for each probability for the first scenario. The values for the same cells in the second scenario are shown in each second line. If two adjacent bars are equal that means all cells of a certain probability from scenario 1 are also probably being attacked in scenario 2. If two adjacent bars are not equal then the second one is shorter and that means that not all cells attacked in scenario 1 are also probably to be attacked in scenario 2.

Let's take as an example the histogram from Figure 9. In the first group of bars (the group that has a small green square on the left side) the first bar shows the number of cells with the value of probability 1 in the first scenario. The second line of the group shows how many of the cells, which appear with value 1 in the first cumulative simulation, are simulated as being attacked in the second scenario and with what probabilities. The second line has lots of colours meaning that some of the cells with value 1 in the first scenario are going to be attacked with the same probability in the second scenario (green cells) or with greater values of probability (represented by cyan, blue, red and yellow parts of the bar) or are not probable to be attacked at all (the black part of the bar).

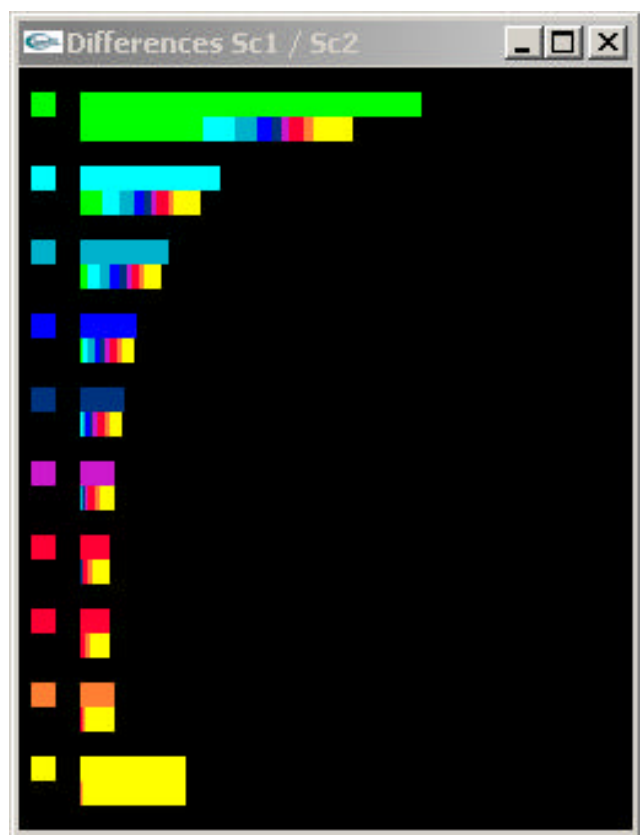

Figure 9: Histogram of differences 


\subsection{Technical considerations}

VICO is developed using Visual $\mathrm{C}++$ 6.0, OpenGL and FLTK on Windows 2000.

The cumulative simulation files generated by SELES are ARC ASCII files. The information in files is coded according to the probability of each cell to be attacked. Each file has $1550 * 1498$ cells encoded as 0 through 10 (see Figure 10).

\begin{tabular}{|l}
\hline 1100101211010109776741000000340 \\
$\ldots \ldots \ldots \ldots \ldots \ldots \ldots \ldots \ldots \ldots \ldots \ldots \ldots \ldots \ldots \ldots \ldots \ldots$ \\
333344400007775688888893449910
\end{tabular}

\section{Figure 10: Example of coding in SELES ARC ASCII files}

\section{Advantages of VICO}

VICO is one of the few tools to implement visual comparison between huge files and the only one to facilitate comparison between MPB simulations. Images in VICO are displayed with no overlapping, having the possibility of seeing both scenarios as they are and also to distinguish the differences between them.

Colours are easy to distinguish and are grouped by colour family. Green, cyan, light blue, blue, dark blue are coding low values of probability (1 through 5), while pink, light red, dark red, orange and yellow are coding high values of probability (6 through 10 ).

The comparison window containing a histogram of frequencies and probabilities that are drawn in parallel facilitates the comparison task. Because the histogram is represented in $2 \mathrm{D}$ the length of the bars are easy to compare.

\section{Future work}

A number of future improvements to this technique are possible.

In the actual implementation there is a legend of the colours used in the scenarios images. It would be interesting to have a legend for the map image according to the type of map used. Example: for an inventory type group map a legend of the colours and species, for an elevation map a legend of colours and elevation in metres.

VICO has the ability to compare two cumulative simulations. It would probably be useful to be able to compare a higher number of scenarios.

There are shown only small areas of the images of the scenarios simulated. Depending if the user finds this useful or not a large window showing the entire image of the cumulative simulations can be added in order to see the context.

We would like to extend our area of research from graphics to databases and to organize the information in a spatial database instead of ARC ASCII files. This will increase the execution speed of our application and it will make it more flexible.

\section{Acknowledgements}

The authors would like to thank Andrew Fall, Gowlland Technologies Ltd., for suggesting this topic of research and for providing SELES simulations files for tests.

\section{References}

[1] Gennady Andrienko, Natalia Andrienko. Descartes System - Interactive Intelligent Cartography in Internet, ERCIM News No.34 - July 1998, also available at http://www.ercim.org/publication/Ercim News/enw34/andr ienko.html

[2] Gennady L. Andrienko, Natalia V. Andrienko. Interactive Maps for Visual Data Exploration. International Journal Geographic Information Science, vol. 13 (4), June 1999, pp. 355-374, also available at http://ais.gmd.de/and/icavis/

[3] M.S.T. Carpendale, D.J. Cowperthwaite, M. Tigges, A. Fall, F.D. Fracchia. The Tardis: A Visual Exploration Environment for Landscape Dynamics. Proceedings, SPIE Conference on Visual Data Exploration and Analysis VI. Santa Fe, USA, 1999, pp. 110-119,

[4] Andrew Fall, Dave Daust, Don G. Morgan. A Framework and Software Tool to Support Collaborat ive Landscape Analysis: Fitting Square Pegs into Square Holes. Transactions in GIS 5(1): 67-86. Special issue of selected papers from the 4th International Conference on Integrating Geographic Information Systems and Environmental Modeling, Banff, Alberta, 2000.

[5] Andrew Fall, Marvin Eng, Terry Shore, Bill Riel, Don Sachs. Mountain Pine Beetle Audit Project Kamloops Forest District Landscape Model. B.C. Ministry of Forests internal report 2001.

[6] Andrew Fall, Joseph Fall. A Domain-Specific Language for Models of Landscape Dynamics. Ecological Modelling 141(1-3): 1-18, 2001.

[7] Greg Pass, Ramin Zabih. Comparing Images Using Joint Histograms. Multimedia Systems 7(3): 234-240,1999.

[8] Michael Swain and Dana Ballard. Colour indexing. International Journal of Computer Vision, 7(1), pages 1132, 1991

[9] Pacific Forestry Centre, available at http://www.pfc.forestry.ca/entomology/mpb/index e.html

[10] SELES, web page available at http://www.cs.sfu.ca/research/SEED 